\title{
Mechanical actuator for biomimetic propulsion and the effect of the caudal fin elasticity on the swimming performance
}

\author{
A. Apalkov R. Fernández J.G. Fontaine T. Akinfiev M. Armada
}

\begin{abstract}
A B S T R A C T
This paper presents a mechanical actuator for the biomimetic propulsion of swimming devices and the experimental study of the effect of the caudal fin elasticity on the overall performance. The design of the proposed drive allows the DC motor to operate at constant speed, so all the power of the motor is spent only for the motion of the caudal fin. A prototype of the actuator, in which the caudal fin serves as a driving element, is manufactured and tested in both laboratory and natural conditions. The swimming speed, the thrust efficiency and the maneuverability are evaluated for caudal fins with different stiffness. The caudal fin whose rigidity varies relative to both vertical and horizontal cross-section, exhibits the best performance. The achieved results also confirm that the proposed actuator could be of great interest to applications in the field of underwater operation, ocean investigation and environmental protection.
\end{abstract}

\section{Introduction}

The use of traditional propellers as actuators for swimming robots causes considerable damage to the environment. The rotational motion of the propellers may injure or kill fish via shear stress, impact or pressure changes [1] and produces noise which could scare fish and force them to withdraw from their normal habitat. Observations show that a fish in a natural environment can achieve great propulsive efficiency, excellent manoeuvrability and low noise performance through the coordinated motion of its body, fins and tail [2]. These advantages are of great benefit to applications in the fields of underwater operation, ocean development, ocean investigation and environmental protection. Since fish are innate swimmers, it seems to be reasonable to copy a driving element in the form of caudal fin for the motion of swimming robots. For this reason, in recent years, there has been increasing interest in the literature regarding the research on biomimetic robotic fish [3-10]. The biomimetic implementation covers the design of the actuators, shape of body and fins.

The majority of the known biomimetic swimming robots mimic either the carangiform or the anguilliform locomotion. In the carangiform mode, the propulsion through the water is achieved with undulating movements of the tail and the last $1 / 3$ part of the body, while the forward part of the body remains relatively immobile $[11,12]$. In such cases, several motors have to be used [13-16], which complicates the design and creates optimisation control problems. During anguilliform locomotion, the whole body participates in large amplitude undulations and since at least one complete wavelength of the propulsive wave is present along the body, lateral forces are adequately cancelled out, minimizing any tendencies for the body to recoil $[11,17]$. Although biomimetic fin undulation is difficult to design and implement, some interesting solutions have been proposed [18,19]. Nevertheless, more tests and investigations should be conducted in order to improve the existing designs in terms of compactness, easier waterproofing and energysaving fin motion. It is also important to note that in many of the designed robotic fish $[20,21]$ the motor used in the actuators works in a back-rotational mode. It is well known that the motor, which operates in a start-stop regime, has low efficiency since a substantial part of the energy is spent on the acceleration and braking of the rotor, gearbox and transmission components. This factor could drastically limit the robot's autonomy.

The work presented in this paper introduces a mechanical drive on the base of a caudal fin and addresses the effects of the caudal fin elasticity on the propulsion performance. The proposed mechanical drive enables to increase the swimming speed and notably reduce the energy consumption, but also moves the caudal fin with high frequencies (up to $26 \mathrm{~Hz}$ ). In almost all the cases cited in the literature, the frequency of the fins' movement is approximately equal 
to the frequency of the natural behaviour of the fish (around $1 \mathrm{~Hz}$ ) $[20,22]$. Therefore, the proposed mechanical drive let us accomplish studies of other parameters such as the thrust force in these unusual conditions. On the other hand, several materials were utilised in order to produce and evaluate experimentally different samples of caudal fins, achieving at the end a very efficient design that is characterised by a variable stiffness. The tests were carried out in both laboratory and natural conditions, using a mechanical prototype of the actuator, in which the caudal fin serves as a driving element.

The rest of the paper is organised as follows. Section 2 explains the design of both the mechanical drive and the caudal fin. Section 3 describes the experiments that were carried out both in laboratory and natural conditions to evaluate and compare the behaviour of the different types of manufactured caudal fins. Some discussions are also presented in this section. Finally, Section 4 summarises major conclusions.

\section{Mechanical drive and caudal fin design}

General view of the mechanical drive is shown in Fig. 1. It consists of an electric motor installed in the robot's body, a pulley coupled to the motor shaft, two cardan joints, the first one connecting a shifted axis located on the pulley with a metallic rod and the second one connecting the metallic rod with a lever, a hinge linking the rotational axis of the lever with the robot's body by means of one of its metallic leaves and finally a caudal fin mounted on a vertical bar, which, in turn, is also fixed to the second metallic leaf of the hinge. The lever has several holes to change the connecting point of the second cardan joint. Fig. 2 illustrates the scheme of the mechanical drive with all its elements. Note that in the neutral position, the caudal fin is aligned with the axial body displacement. With this configuration, at one motor revolution, the caudal fin completes a full cycle, passing twice through the neutral position.

The maximum opening angle of the caudal fin peduncle, $\alpha / 2$, measured from the neutral position, varies depending on the location of the point where the lever connects with the second cardan joint. The closer this connection point is to the hinge, the larger will be the magnitude of $\alpha / 2$. The magnitude will decrease as the location of the connection point moves away from the hinge. The design of the lever with several holes allows us to change the connecting point of the second cardan joint, and consequently, modify the maximum opening angle of the caudal fin peduncle. These holes will limit in both number and magnitude the maximum opening angles that can be selected. Therefore, the opening angles that could be utilised are tightly related with the design of the mechanical drive. To eliminate the misalignment that may occur with the change of the cardan position, the drive is provided with the possibility of changing the position of the electric motor on the base.

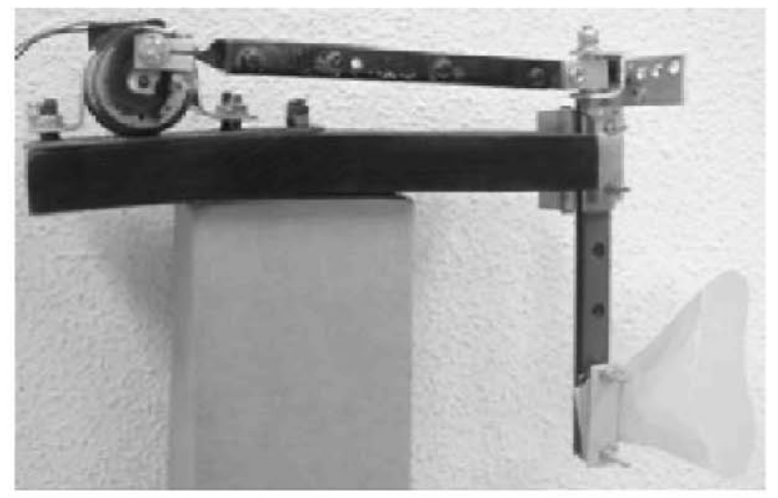

Fig. 1. General view of the mechanical drive.
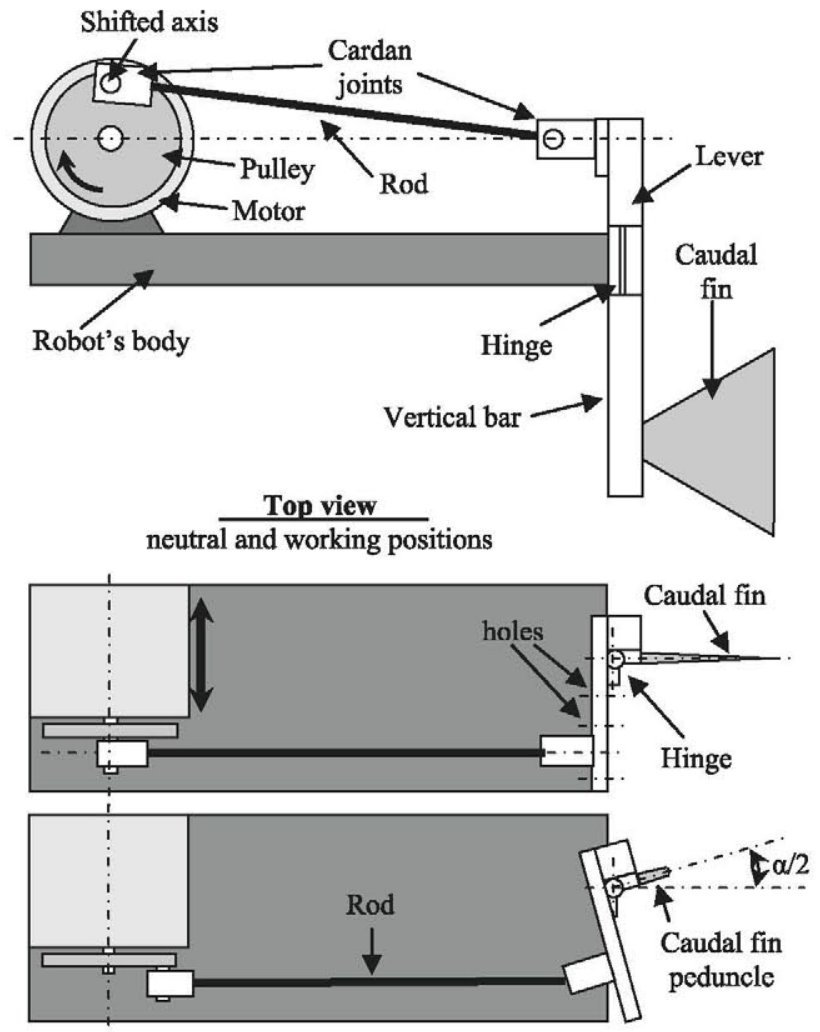

Fig. 2. Scheme of the mechanical drive.

In the proposed drive the electric motor rotates at constant speed. This mode of operation is the most economical, because all the motor power is used for the motion of the caudal fin and only a minimum percentage of power is wasted in overcoming the friction in the cardan joints and the hinge. The motion frequency of the caudal fin can be easily varied by changing the voltage supplied to the electric motor.

In order to design an efficient caudal fin and to obtain a better knowledge of how different materials affect its performance, an experimental study is carried out. The selected materials and their dispositions will determine the characteristic stiffness of the caudal fins, which is the feature that finally will impact in the propulsion performance. For the proposed study, four operating members in the form of a fishtail with different stiffness characteristics are made. One of these operating members (type A) is made in such a way that the stiffness decreases simultaneously from the fixed end towards the free end and from the top and bottom edges to the middle. The other types of operating members (B, C, D) have a stiffness that virtually does not change as a function of the vertical coordinate, but decreases from the fixed end to the free end. In this series, each type is more rigid than the previous one, so type D is almost absolutely rigid. Fig. 3 shows the four types of caudal fins.

Each caudal fin is composed of a central plate, which forms the basis and provides the shape to the caudal fin, and one or more pairs of reinforcing ribs that are glued to the central plate. Fig. 4 shows a general scheme of the manufactured caudal fins. Note that starting from the central plate, a pair of reinforcing ribs are added, one on each side of the central plate. Next a new pair of reinforcing ribs is glued over the ribs that were placed previously. This process can be repeated until completing a maximum of three pairs of reinforcing ribs, as in the case of the A type. Polyester and two different kinds of flexible plastic were used for the manufacture of both central plates and reinforcing ribs. The first type of plastic (I) has an elastic modulus of $E=4.1 \times 10^{3} \mathrm{MPa}$ while the second (II) is more elastic, 


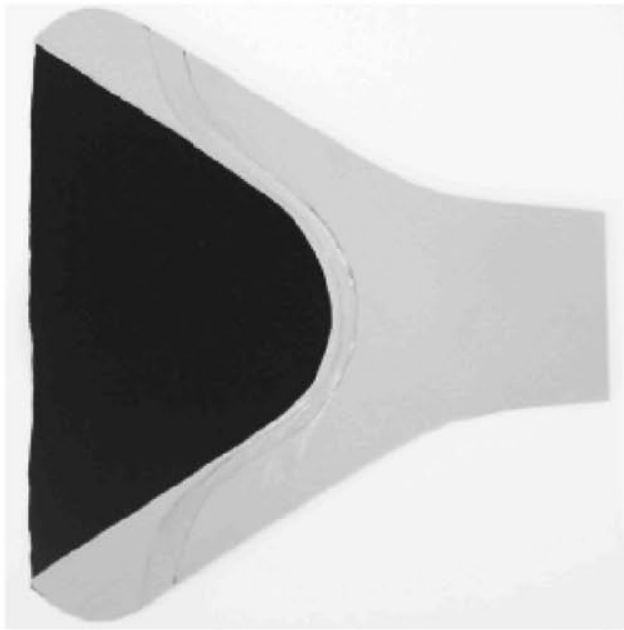

(a)

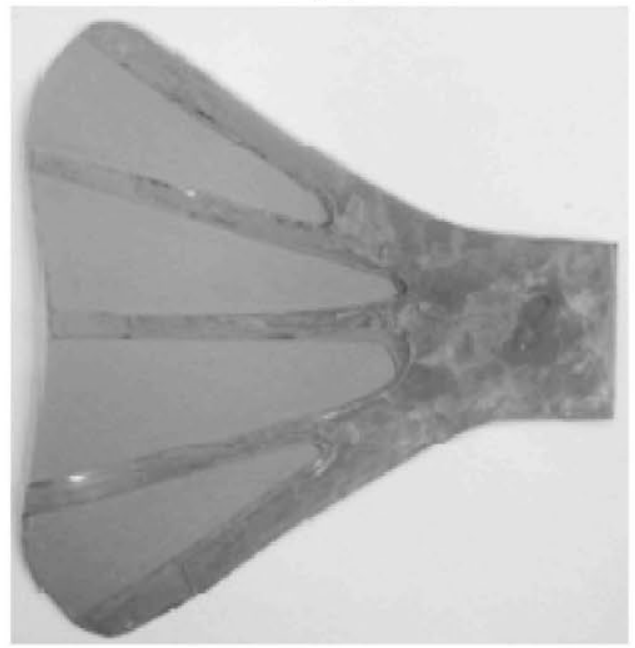

(c)

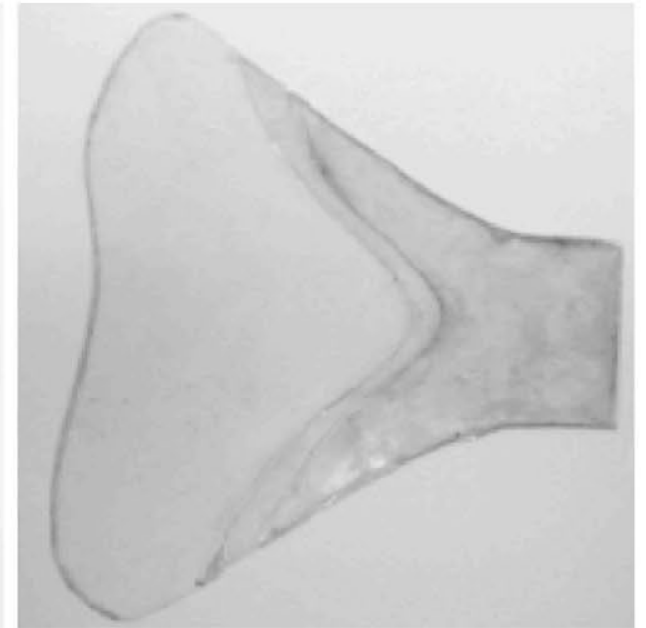

(b)

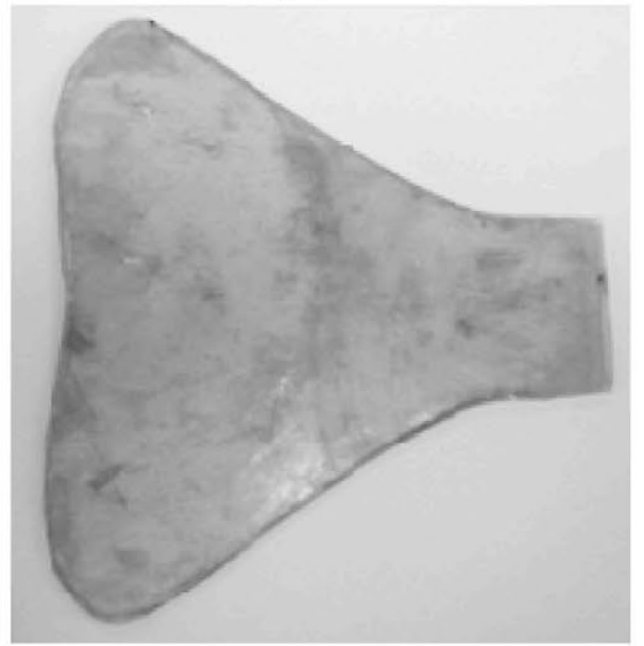

(d)

Fig. 3. Manufactured caudal fins. (a) Type A. (b) Type B. (c) Type C. (d) Type D.

with a modulus of $E=2.8 \times 10^{3} \mathrm{MPa}$. The dimensions $(a, b)$ of the central plates and the size $(c, d, e)$ of the reinforcing ribs are also depicted in Fig. 4. The shaded part of Fig. 4 shows the area used to fix each caudal fin in the mechanical drive. Table 1 summarises the characteristics of each type of manufactured caudal fin, describing the materials used, the number of pairs of reinforcing ribs added, and their dimensions. The magnitude of the variable findicates how the actual thickness of the caudal fin changes as the reinforcing ribs are added. This magnitude is affected not only by the sum of the thickness of the central plates and the reinforcing ribs but also by the amount of glue needed to paste them.

Thus, in the caudal fin of type A, the central plate was fabricated with polyester and three reinforcing ribs of different shapes were glued on each side of the central plate. As we mentioned previously,

Table 1

Characteristics of the manufactured caudal fins.

\begin{tabular}{|c|c|c|c|c|c|c|c|c|c|}
\hline Type of caudal fin & Structure & No. of plate/pair of rib & Material & $a[\mathrm{~mm}]$ & $b[\mathrm{~mm}]$ & $c[\mathrm{~mm}]$ & $d[\mathrm{~mm}]$ & $e[\mathrm{~mm}]$ & $f[\mathrm{~mm}]$ \\
\hline \multirow{4}{*}{ A } & 1 central plate & 1 & Polyester & 95 & 100 & - & 95 & - & 0.08 \\
\hline & & 2 & & - & - & 95 & 38 & 13 & 0.6 \\
\hline & 3 pairs of ribs & 3 & II & - & - & 76 & 37 & 12 & 0.95 \\
\hline & & 4 & & - & - & 69 & 36 & 11 & 1.35 \\
\hline \multirow{3}{*}{ B } & 1 central plate & 1 & & 95 & 100 & - & 90 & - & 0.15 \\
\hline & 2 nairs of ribe & 2 & I & - & - & 64 & 34 & 15 & 0.48 \\
\hline & 2 pairs of ribs & 3 & & - & - & 54 & 27 & 9 & 0.84 \\
\hline \multirow{4}{*}{ c } & 1 central plate & 1 & II & 95 & 100 & 85 & $50 / 35$ & $7 / 5 / 5 /$ & 0.15 \\
\hline & & & & & & & $35 / 50$ & $5 / 7$ & \\
\hline & $\begin{array}{l}2 \text { pairs of } \\
\text { reinforcing plates }\end{array}$ & 2 & I & - & - & - & - & - & - \\
\hline & & 3 & - & - & - & - & - & 0.84 & \\
\hline D & 9 plates & $1-9$ & I & 95 & 100 & - & 87 & - & 1.6 \\
\hline
\end{tabular}




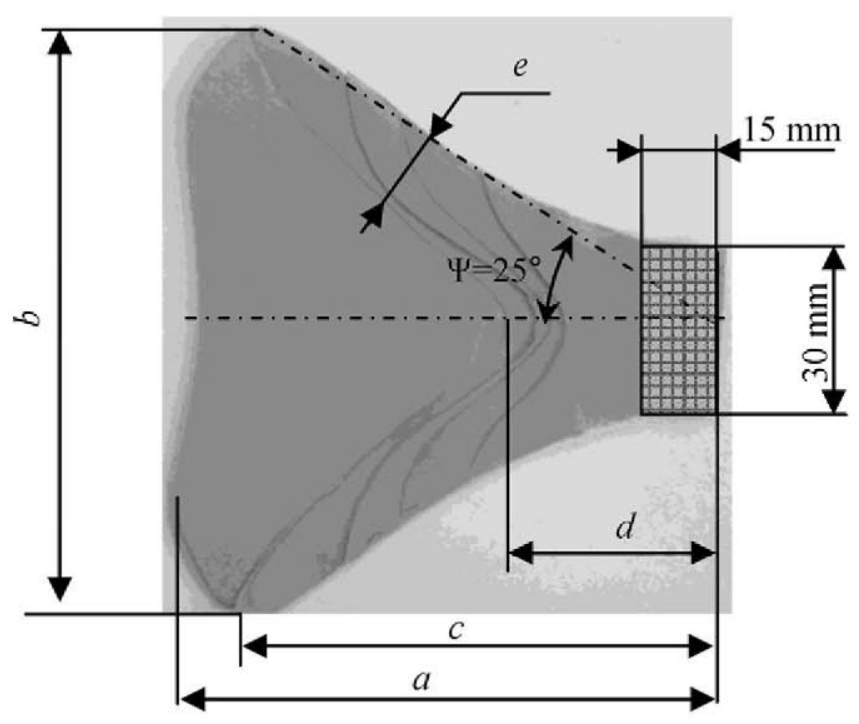

Fig. 4. Scheme of the manufactured caudal fins.

this configuration produces the rigidity of the caudal fin to vary in both horizontal and vertical directions. The caudal fin of type B consists of one central plate made with the first plastic type (I) and two reinforcing ribs of the same material mentioned before. The caudal fin of type $C$ consists of one central plate manufactured with the second plastic type (II) and two reinforcing plates glued on each side of the central plate. Each of these four plates, manufactured with the first plastic type (I), has 5 stiffeners, two on the extremes and three on the central part. The extreme stiffeners have a width of $7 \mathrm{~mm}$, and the central ones a width of $5 \mathrm{~mm}$. With this configuration, that is illustrated with more details in Fig. 5, the resulting caudal fin is elastic, but actually, the entire surface has a constant and fixed rigidity.

On the other hand, the structure of the caudal fin of type D differs from the rest. This caudal fin is the only one that does not have reinforcing ribs and consists entirely of 9 plates of identical shape, made with the first type of plastic (I), and glued together.

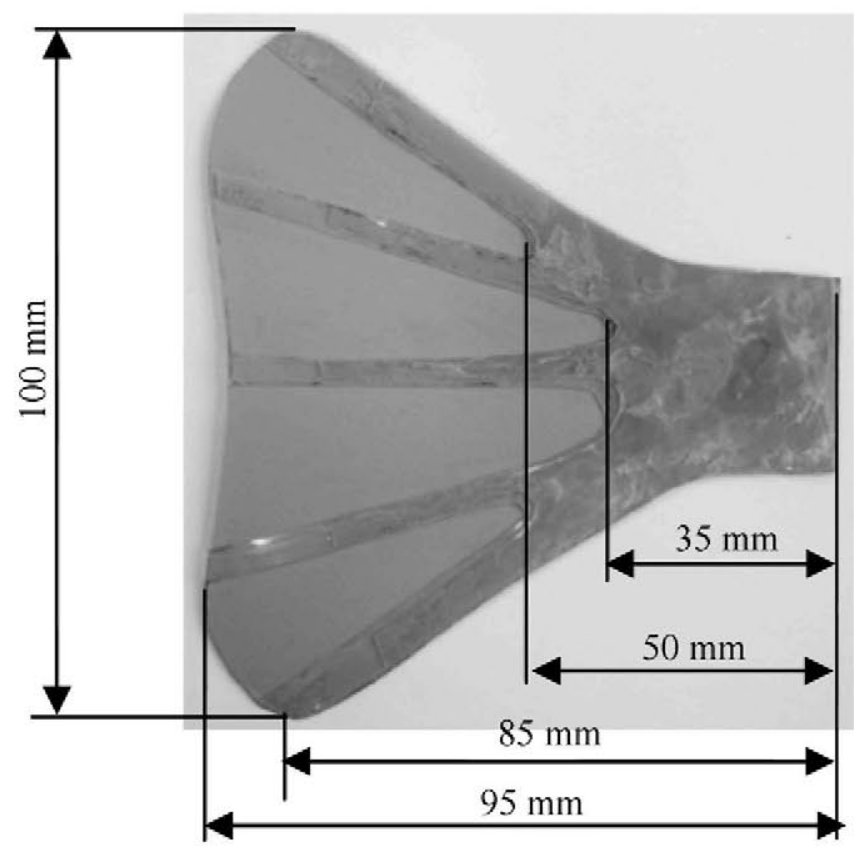

Fig. 5. Scheme of the c type caudal fin.
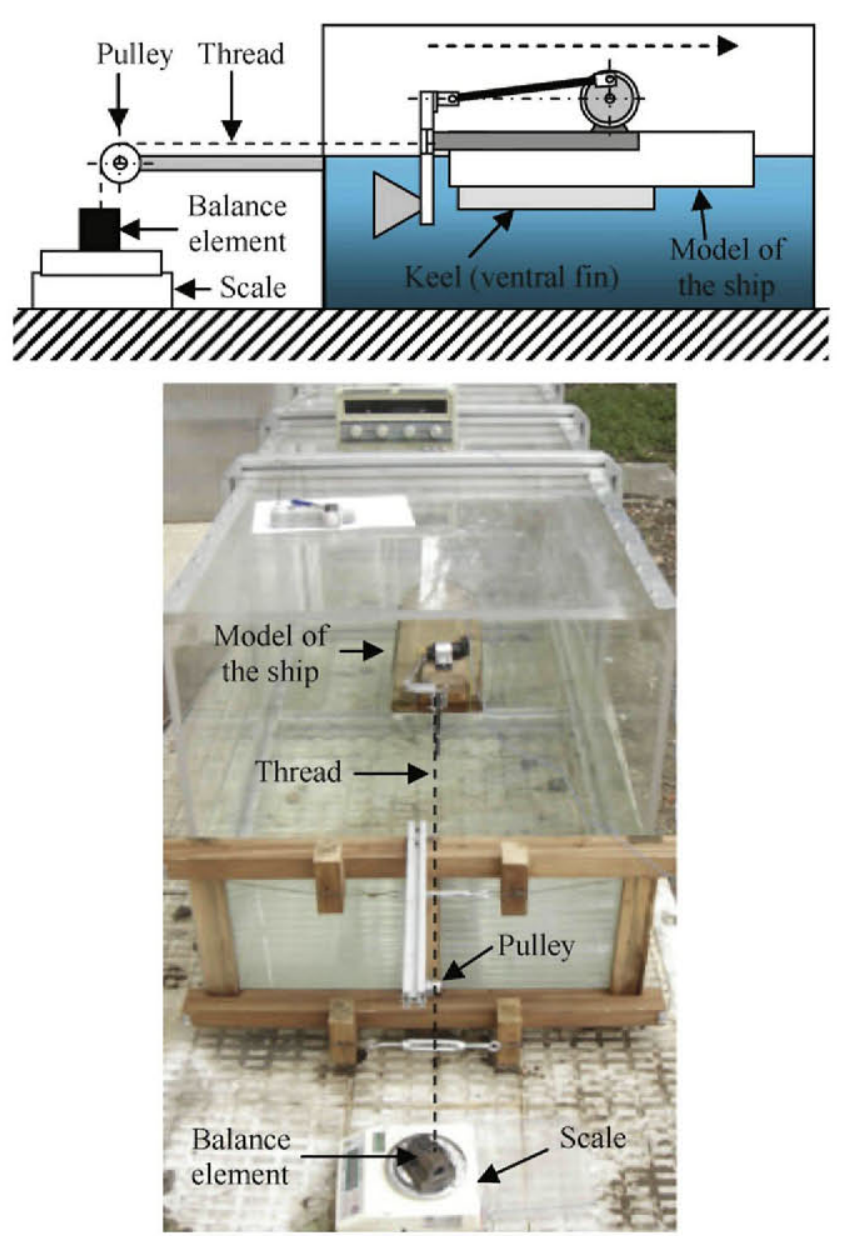

Fig. 6. Testbed set-up.

Therefore, this type of caudal fin turns out to be very inflexible and does not present changes in the stiffness in both horizontal and vertical directions.

\section{Experimental evaluation}

To evaluate the performance of the proposed actuator with the different types of caudal fins, various experiments were carried out in both laboratory and natural conditions.

\subsection{Laboratory tests}

To simulate the field conditions in a lab controlled environment, a testbed was designed and implemented (see Fig. 6). It consisted of a tank, a model of the swimming device where the proposed actuator was installed, and a balance element. Since for the field experimentation stage a small ship had been selected as a swimming-on-surface device, a model of this small ship was utilised (see Fig. 7). Nevertheless, note that this model could also represent the robotic fish's body or any other swimming device. The physical dimensions of the built tank were $750 \mathrm{~mm} \times 750 \mathrm{~mm} \times 2100 \mathrm{~mm}$. The walls of the tank were constructed from Plexiglass, enabling visual inspection of the ship model. The tank was filled with water until a depth of $300 \mathrm{~mm}$. The model of the ship was located inside the tank and connected to a balance element by means of a nylon thread. In order to reduce the influence of the friction at the inflection, the thread was passed through a pulley with ball bearing. The balance element rested on a scale in order to measure and record its mass during the 


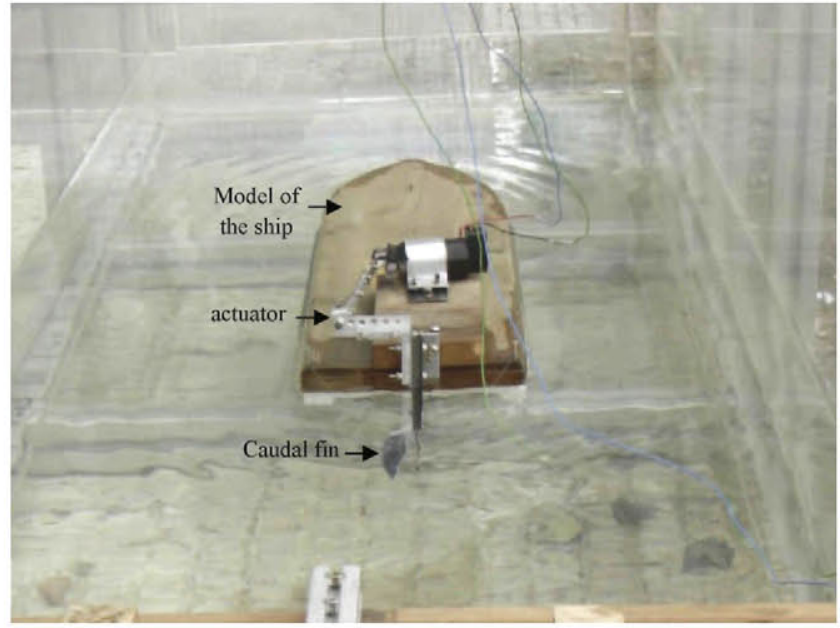

Fig. 7. Actuator installed in the model of a small ship.

experiments. When the motor was energised, the caudal fin started its motion and consequently, the ship began to move forward as much as the free length of the nylon thread allowed it to. After that, the nylon thread was pulled, and the load mass of the balance element on the scale decreased. The difference in the weight of cargo was equal to the tension force on the nylon thread. In the course of the experiment the value of the voltage $(U)$ supplied to the motor, the amperage $(I)$ and the frequency $\left(f_{r}\right)$ of motion of the caudal fin were fixed.

Throughout the laboratory experiments, the dynamics of water circulation during the oscillation of the caudal fin was observed. In the movement of a rigid caudal fin (such as the D type), the mass of water is distributed in all directions, i.e. in the both directions, $X$ and $Y$, as shown in Fig. 8. As can be seen, the rigid model pushes the water in all directions, although the main function of caudal fins should be the movement of water along the $X$-axis.

When the motion of an elastic caudal fin (such as the B type) is produced, a push from the water occurs, and consequently an increase of traction takes place (see Fig. 9). This happens because the stiffness of the elastic caudal fin is changing in the $X$ direction. However in this case, there is also a disadvantage. As in the rigid model, a part of the water mass is scattered in the $Y$ direction, because the stiffness of the caudal fin in this direction is not changed and the edge of the caudal fin remains straight.

At low frequencies, the caudal fins oscillate as shown in Fig. 10(a), which we will call first form of oscillation. With an increment of the tail-beat frequency, the form of fluctuations varies, forming larger oscillations, as shown in Fig. 10(b). It was observed
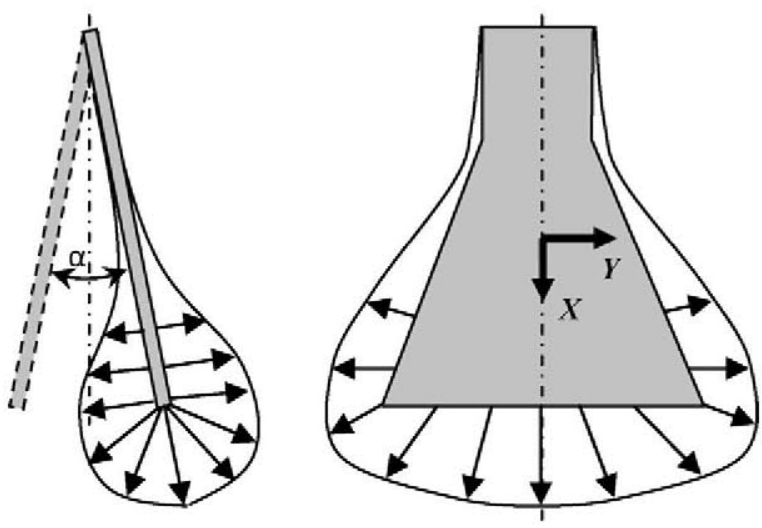

Fig. 8. Distribution of the mass of water for the motion of a rigid caudal fin.
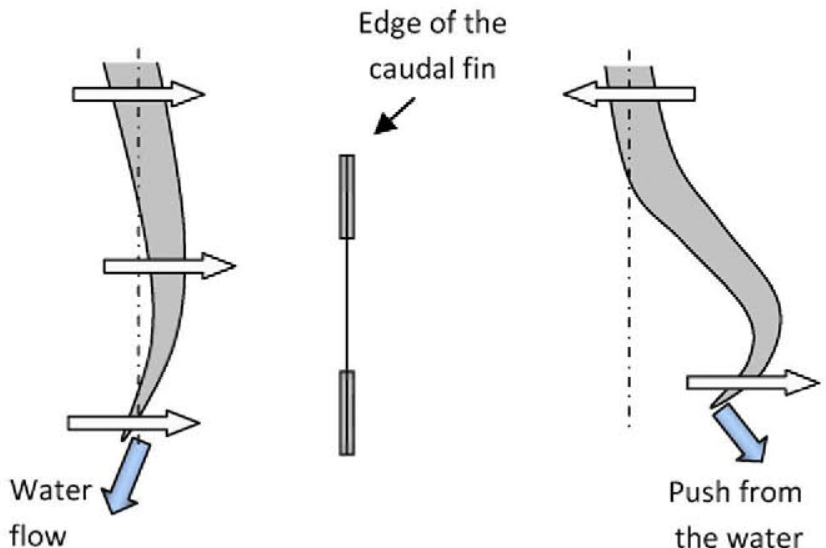

Push from

the water

Fig. 9. Motion dynamics of flexible devices.

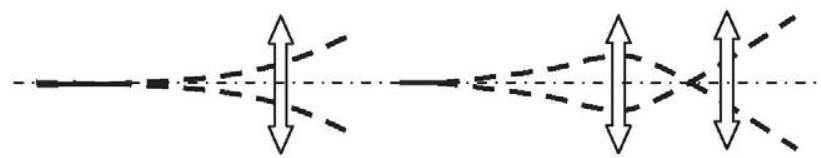

(a)

(b)

Fig. 10. Forms of oscillations: (a) First form. (b) Second form.

that an intermediate form of oscillation of the central plate precedes (Fig. 11) a transition to the second form of the oscillation (Fig. 10(b)).

Thus, during the laboratory studies, a most efficient design of caudal fin was developed. Moreover during the preparation of the different types of caudal fins it was found that two factors have to be taken into account:

- The central plate must be made from an elastic material. For sample A, polyester was used for the central plate, while reinforcing ribs were made of elastic plastic of type II. In this case, a "water flow" is created (see Fig. 12) because the edge of the caudal fin becomes convex in shape, and the lateral ribs are inclined and do not allow water flow to move in the $Y$ direction.

- Caudal fin should have a variable stiffness. In this way, the model pushes against the water, which helps to increase the traction force.

Caudal fin of type A was made taking into account these two factors and showed to be the best of all the tested caudal fins.

In the first phase of laboratory tests, the characteristics of the four manufactured caudal fins (Table 1) were studied. Each experiment was repeated ten times in order to obtain the mean values that are plotted in the all the graphs. Fig. 13 shows the plots of the tension force of the nylon thread (thrust force) depending on the applied power and the tail-beat frequency. It is important to note that before beginning the study of the different parameters of interest, we experimentally determine the range of tail-beat frequencies that can be used without experiencing a failure in each caudal fin. Thus, for each caudal fin, the tail-beat frequency was increased until the failure occurred. These limit frequencies were used in the lab experiments carried out. To obtain the results shown in Fig. 13, the mechanical drive was adjusted to provide a maximum opening angle of $21^{\circ}$. Quite interesting is the case of the sample D. Observe that for this rigid sample, as the applied power increases, there is a slight increase of the tension force. However, at the applied power of $5.5 \mathrm{~W}$, caudal fin moves to the second form of oscillation and as the voltage applied to the motor increases, the useful force and the 


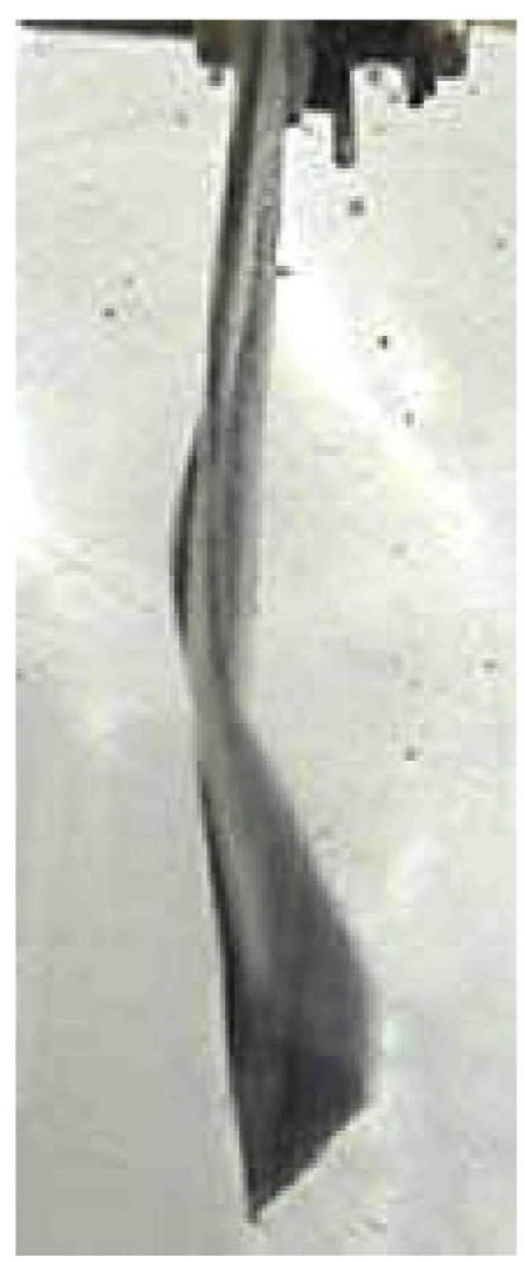

Fig. 11. Intermediate state of the working element.

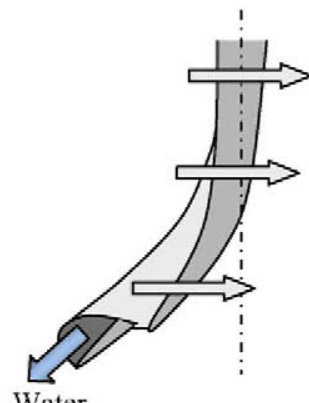

Water

flow
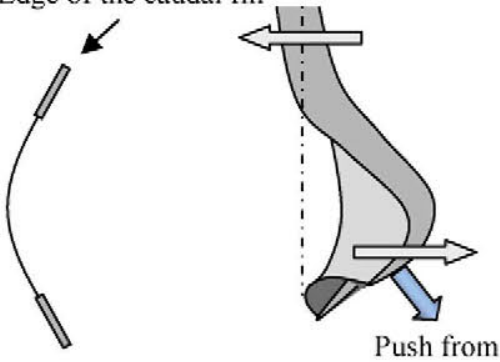

the water
Edge of the caudal fin ,
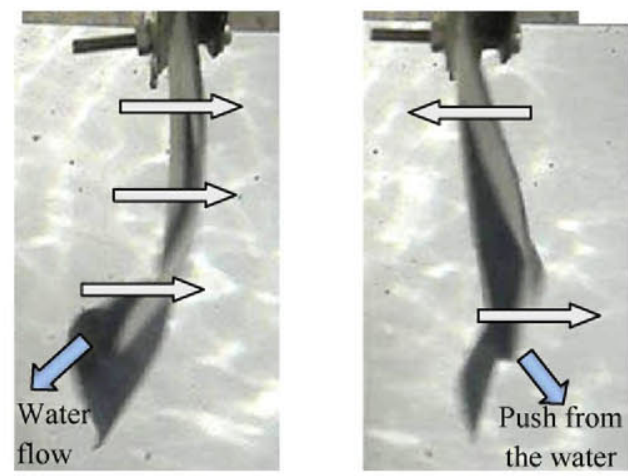

Fig. 12. Working state of the caudal fin.
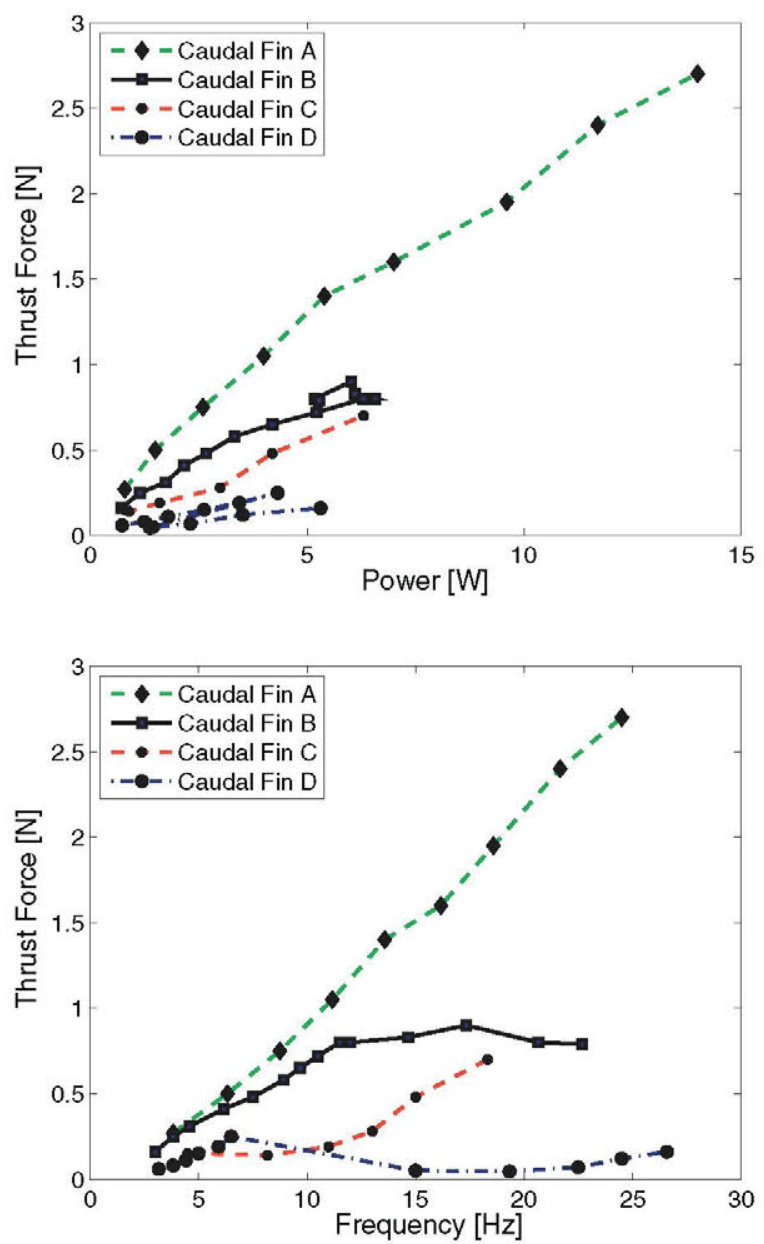

Fig. 13. Thrust force as function of the applied power and the tail-beat frequency for an opening angle of $21^{\circ}$ of the caudal fin peduncle.

power fall. Further increase in voltage leads to a slight increase in the useful power with an appreciable increase in applied power.

Similar patterns are observed for the elastic model B. The shift to the second form of oscillation occurs when a power of $6.5 \mathrm{~W}$ is applied. This process is accompanied with an increased thrust force while the power supplied is reduced. Then, the power together with the thrust force, fall simultaneously, and after that, the power begins to rise again. Thus, a "loop" is formed in the graph. Furthermore, in this case, during the transition to the second form of oscillation, the caudal fin beat frequency grows almost linearly. For samples $A$ and $C$ the shift to the second form of oscillation was not observed. Thus, such a transition to the second and subsequent modes of oscillation occurs at higher frequencies. The plots of the dependence become nearly linear. It is clearly seen that sample A, by its characteristics, is 2 times more efficient than sample $C$.

Fig. 14 shows the plots of the relationships between the thrust force, the applied power, and the tail-beat frequency for a maximum opening angle of $27^{\circ}$. The same behaviours that were previously studied are observed now for the new opening angle. Let us consider the characteristics of sample B in Figs. 13 and 14. It can be seen that the smaller the opening angle, the faster the caudal fin shifts to the second form of oscillation with both a lower thrust force and a lower applied power. It should be noted that the shift onto the second form of oscillation in both cases occurs at a frequency of about $15 \mathrm{~Hz}$, so, in the aquatic environment, a second form of oscillation is most likely for this particular sample of caudal fin [23]. Switching to another form of oscillation will be accompanied by a loss of both thrust force and applied power. Studies were 

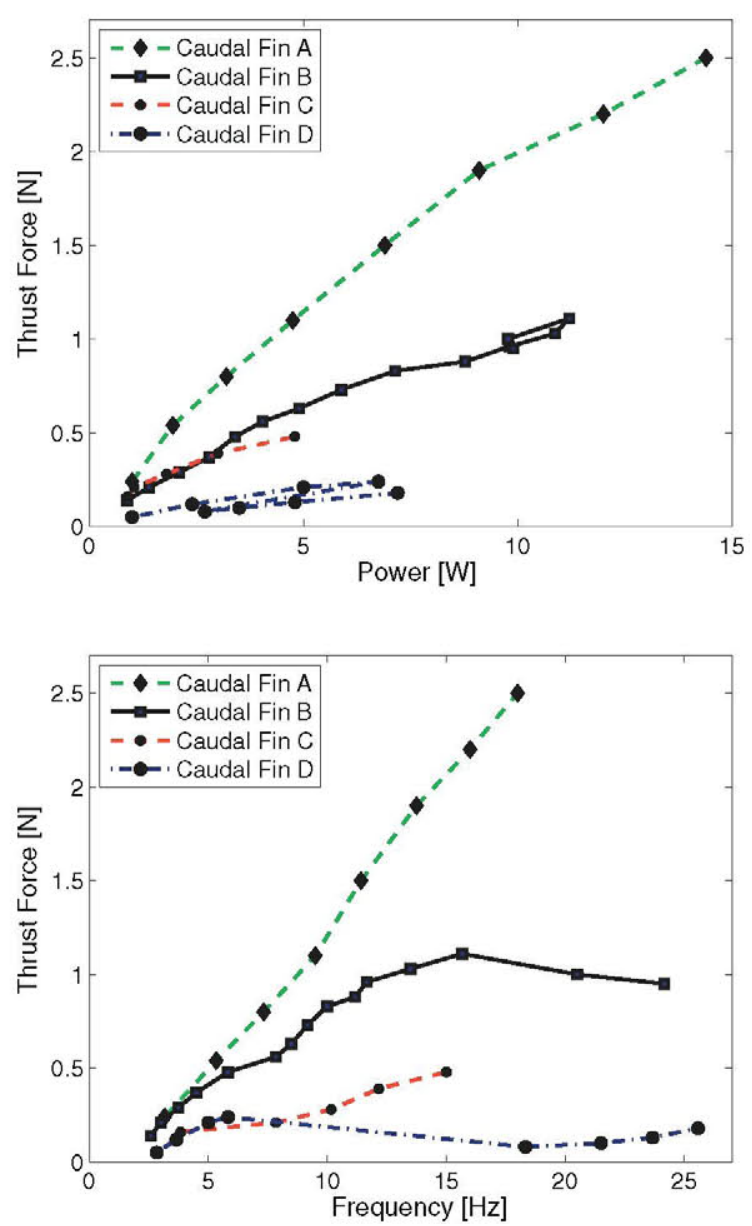

Fig. 14. Thrust force as function of the applied power and the tail-beat frequency for an opening angle of $27^{\circ}$ of the caudal fin peduncle.

carried out for different opening angles: $18^{\circ}, 21^{\circ}, 27^{\circ}$ and $37^{\circ}$. In this way, the most effective type of caudal fin was found experimentally and it turned out to be the sample of type A. In this type of caudal fin, the stiffness decreases from the end fixed to the drive to the free end, and at the same time, it varies from the top and bottom edges to the middle part. Moreover, polyester was used for the manufacture of its central plate (Fig. 15(a)). We used this material, because during testing it was found that the central plate was affected by high cyclic loads, which led to the failure of the central plate in areas of stress concentration, i.e. in places with absence of the additional lateral reinforcing ribs (Fig. 15(b)). In this regard, we decided to use Polyester in the central plate, in order to increase the workloads without experiencing a failure of the working element. As a result, this type of caudal fin showed the best performance of all the tested caudal fins.

In the next stage of this work, the optimal opening angle for the caudal fin of type A was determined experimentally. So, a caudal fin of type A was selected as the working element. Fig. 16 shows the relationship between the thrust force, the applied power and the tail-beat frequency, for different opening angles.

The figure shows that the larger the opening angle is, the more power must be applied. When the opening angle is $37^{\circ}$, an increase in the thread tension force is accompanied by a noticeable increase in power. With a reduction of the opening angle, it is possible to achieve the same values of thread tension by applying a lower power. With an approximate aperture angle of $21^{\circ}$, stabilisation occurs, i.e. for a given caudal fin, the ratio of the useful power versus the applied power is the best. Obviously, a further decrease in the angle would lead to a reciprocal increase of the applied power and a reduction in the thrust force. Note that for an opening angle of $37^{\circ}$, twice the power should be applied in order to get the same thrust force as for an opening angle of $18^{\circ}$. The tail-beat frequency also differs approximately by a factor of 2 .A high repeatability was attained in the measurements shown in Figs. 13,14, and 16. Consequently, a negligible standard deviation of the data was registered.

On the other hand, the peak to peak amplitudes achieved by the tips of the different caudal fins were measured using the frame by frame projections obtained with a video camera running at 50 frames per second. Fig. 17 shows the mean values of the ten measurements recorded at each frequency for each caudal fin with $\alpha / 2=21^{\circ}$. Vertical bars represent the standard deviation. With the exception of the caudal fin of type $\mathrm{D}$, all fins present a reduction in the tail-beat amplitude at high frequencies.

Lastly, in order to determine the amount of noise generated by the caudal fins, a Brüel \& Kjær hydrophone of type 8104 was utilised to acquire their acoustic emissions (see Fig. 18(a)). The mechanical drive was properly isolated, reducing the sounds transmitted to the water. One of the most useful visual representations of audio signals is the sonogram, also called spectrogram, that is a

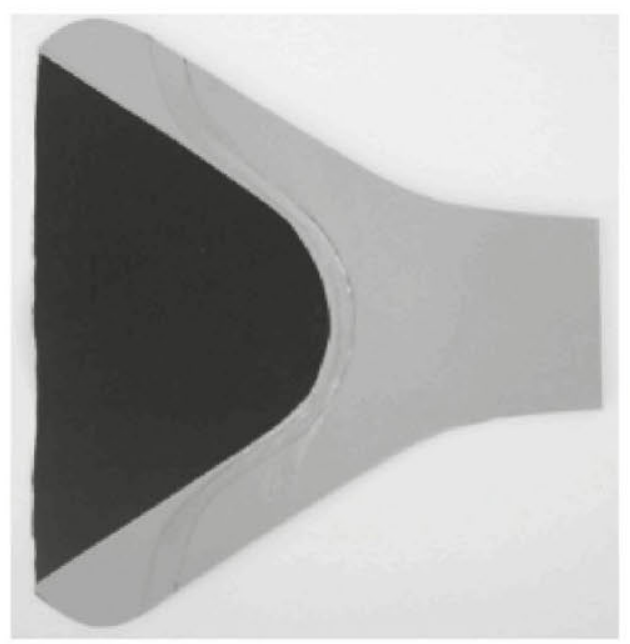

(a)

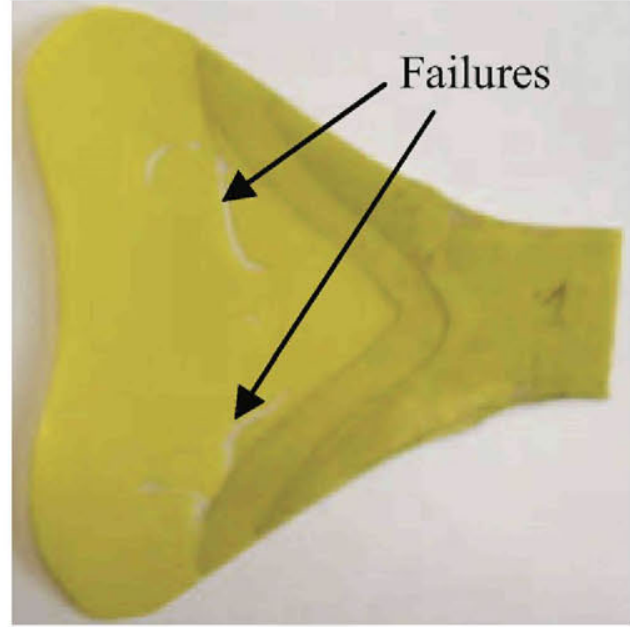

(b)

Fig. 15. (a) Caudal fin of A type. (b) Typical failures in areas of stress concentration. 

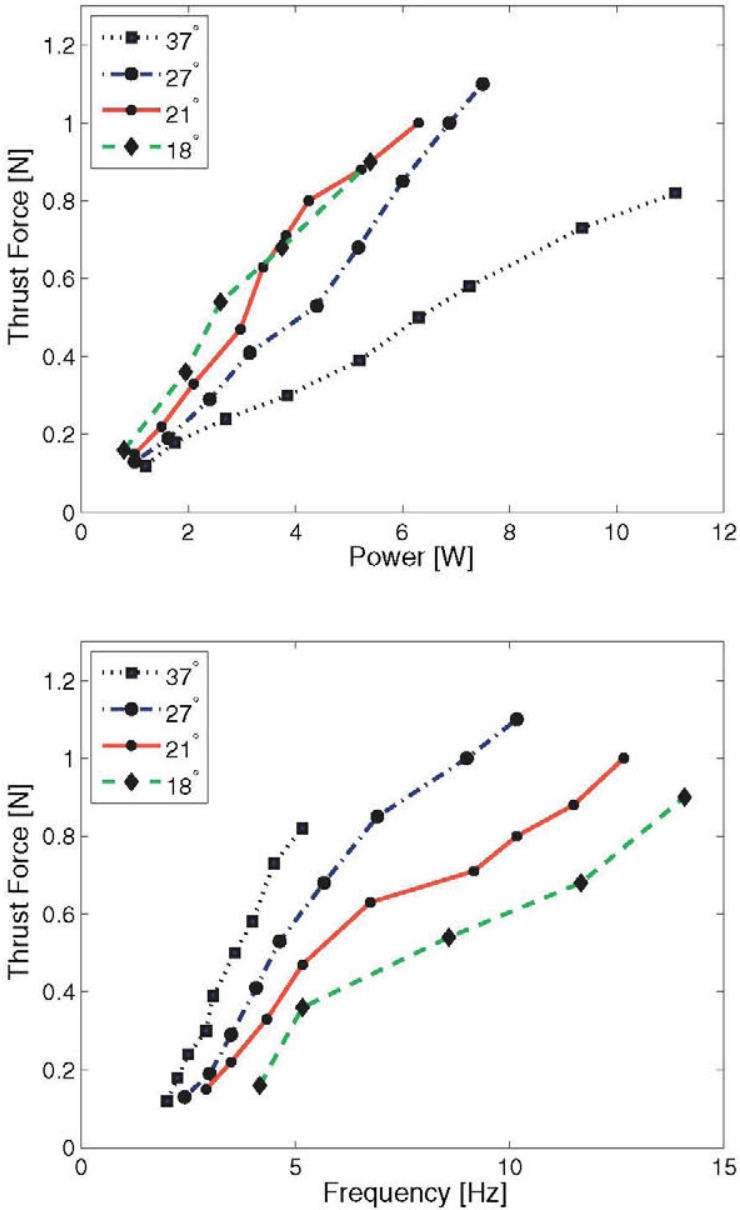

Fig. 16. Relationship between the thrust force, the applied power and the tail-beat frequency, for different opening angles of a Caudal Fin of type A.

colour - or grey - scale rendition of the magnitude of the STFT (Short-Time Fourier Transform), on a 2D plane where time and frequency are the orthogonal axes. Therefore, the power at each time-frequency point is expressed by means of a colour. The resulting spectrogram from the caudal fin of type A is shown in Fig. 19. Then, this result was compared with the acoustic emissions generated by the propeller shown in Fig. 18(b). The propeller has a radius of $10 \mathrm{~cm}$ and during the experiments it was moved by a DC motor rotating with the same velocity that was used for the caudal fin.

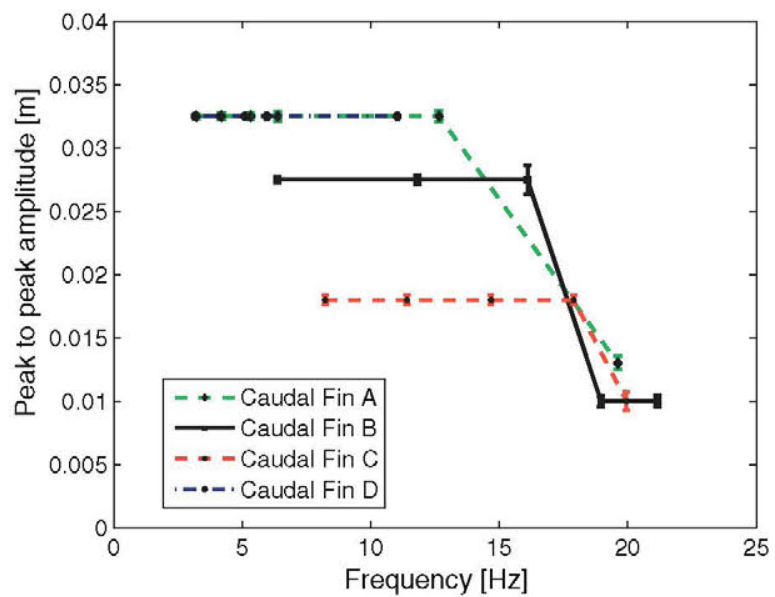

Fig. 17. Peak to peak amplitudes achieved by the tips of the different caudal fins.

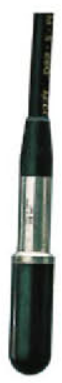

(a)

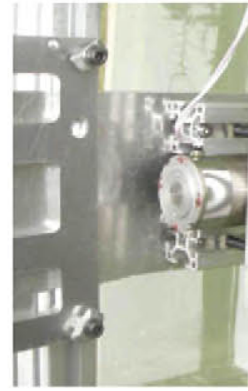

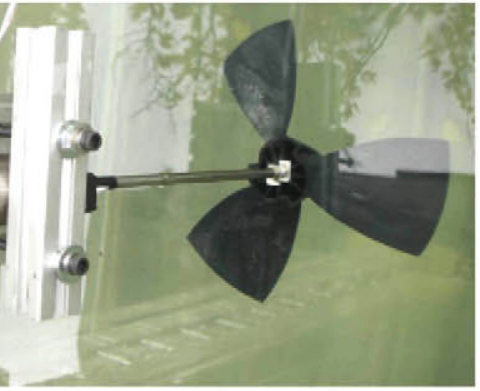

(b)
Fig. 18. (a) Brüel \& Kjær hydrophone - type 8104. (b) Propeller.

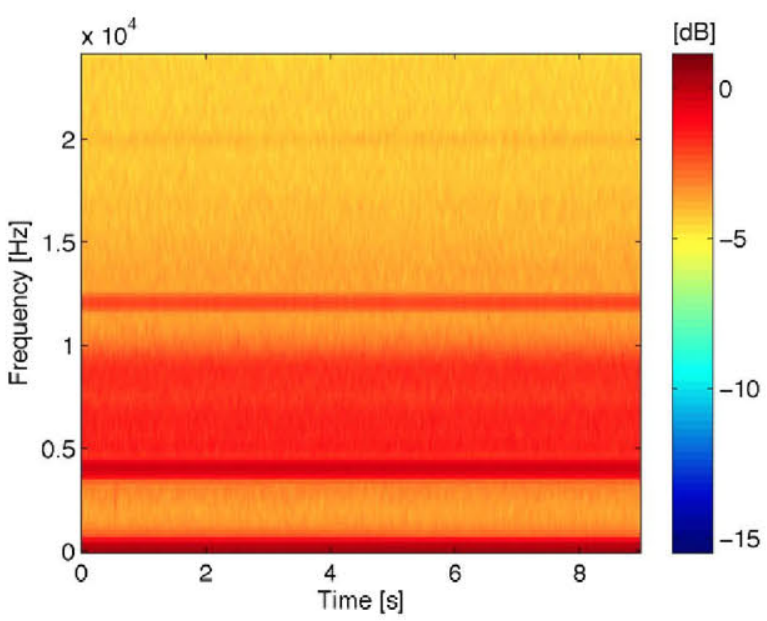

Fig. 19. Caudal fin spectrogram.

Fig. 20 illustrates the spectrogram of the propeller when it is completely submerged in the water. Fig. 21 displays the spectrogram of the propeller when it is partially submerged in the water. As is observed, the caudal fin produces lower noise than the propeller. This is especially true when the comparison is done with the propeller that is partially submerged in water. A primary source of the noise measured in this case is the phenomenon of cavitation where negative pressures occur as the propeller blades rotate, resulting in the formation of bubbles that subsequently collapse with a bang.

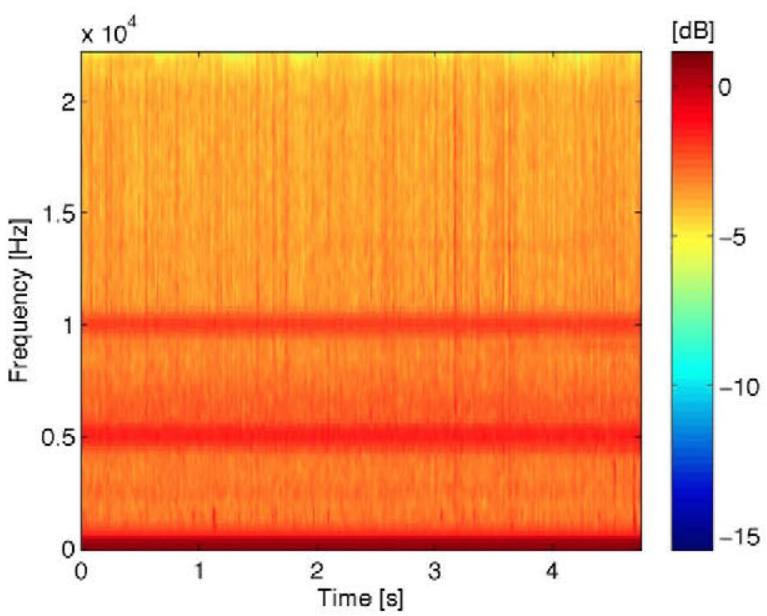

Fig. 20. Propeller spectrogram-the propeller is completely submerged in the water. 


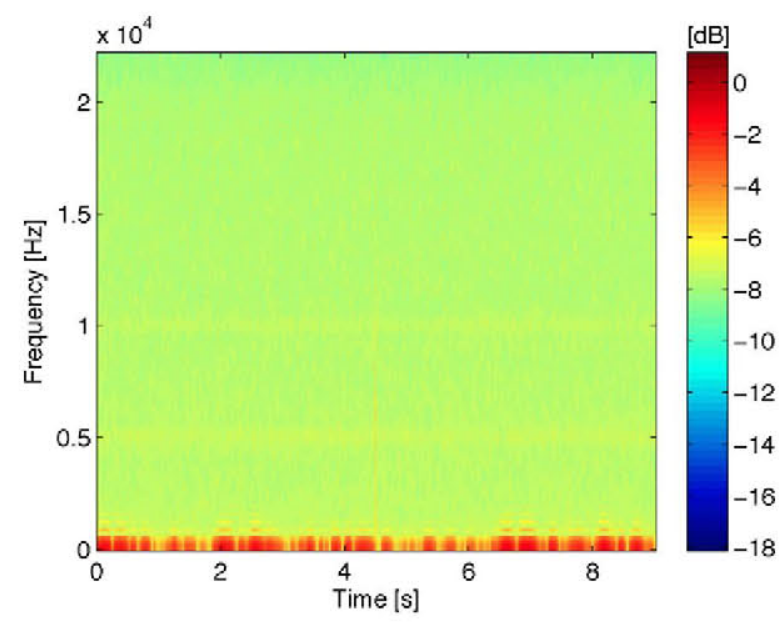

Fig. 21. Propeller spectrogram - the propeller is partially submerged in the water.

\subsection{Field tests}

For a full-scale testing in natural conditions, the designed actuator was installed in a small swimming ship (see Fig. 22). To achieve a rectilinear motion of the ship, a metal keel was placed at the bottom of its hull. This metal keel that acts as a ventral fin has a thickness of $1.5 \mathrm{~mm}$. The mechanical drive was adjusted to provide a maximum opening angle of $21^{\circ}$. The experiments were carried out on the open water surface of a lake. Each experiment was repeated ten times in order to obtain the mean values that are plotted in the following graphs. Fig. 23 displays the velocities attained by the ship with each of the manufactured caudal fins for different tail-beat frequencies. Vertical bars represent the standard deviation. It can be seen that the highest velocities are attained by the caudal fin of type A.

The swimming performance of the ship can be compared with real fish by evaluating the Strouhal number. The Strouhal number is a dimensionless measure of thrust optimality $[24,25]$ and it is given by:

$S t=\frac{f_{S} A_{w}}{U}$

where $f_{S}$ is the tail-beat frequency [Hz], $A_{w}$ is the wake width $[\mathrm{m}]$ (usually approximated as the tail-beat peak-to-peak amplitude), and $U$ is the average forward velocity [m/s]. Typically, thrust increases with increasing Strouhal number, but efficiency peaks near 0.3. Triantafyllou et al. [25] concluded that, in oscillating foils, thrust development is optimal for a specific range of St (namely $0.25<S t<0.4$ ). In their papers, [24,25], Triantafyllou et al. analysed twelve species (dolphins, sharks, some scombroids and other bony fishes) whose swimming kinematics were found in the literature

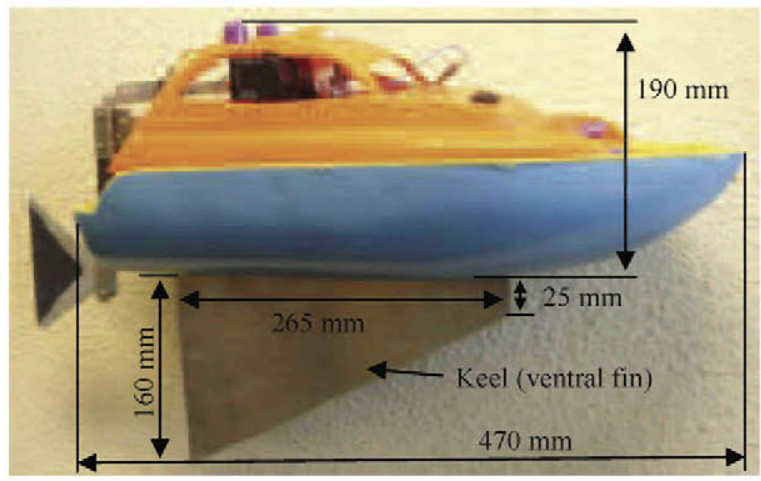

Fig. 22. Prototype of the ship with the proposed actuator.

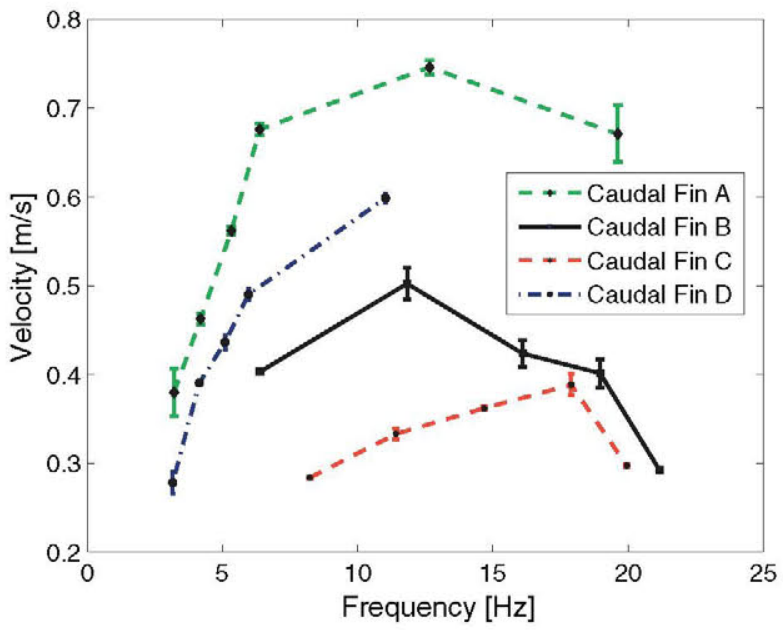

Fig. 23. Swimming speed vs, tail-beat frequency.

and concluded that most of these swimming animals indeed swim in this interval. Then, it has been argued that fish likely evolved to swim in this narrow interval. The Strouhal numbers of the ship using the manufactured caudal fins are collected in Fig. 24. Vertical bars represent the standard deviation. The results are quite different depending on the type of caudal fin. For frequencies lower than $6.5 \mathrm{~Hz}$, the caudal fins of both types, A and D, exhibit a Strouhal number that falls within the optimal range. Moreover, the caudal fin of type A exhibits a Strouhal number of around 0.3, that coincides with the efficiency peak. Therefore, it is possible to conclude that with these types of caudal fins, the performance is in the fast efficient swimming category. This is mainly due to the large tail-beat amplitudes and high speeds achieved with these types of caudal fins. At frequencies higher than $12.5 \mathrm{~Hz}$, the caudal fin of type A switches to its second form of oscillation, which leads to a reduction in the thrust force, while the caudal fin of type D experiences a failure. On the other hand, the Strouhal numbers for the caudal fins of types B and C do not fall within the optimal range, and consequently, the performance is not in the fast efficient swimming category.

Another important parameter is the Froude number [20], given by:

$F r=\frac{U}{\sqrt{g L}}$

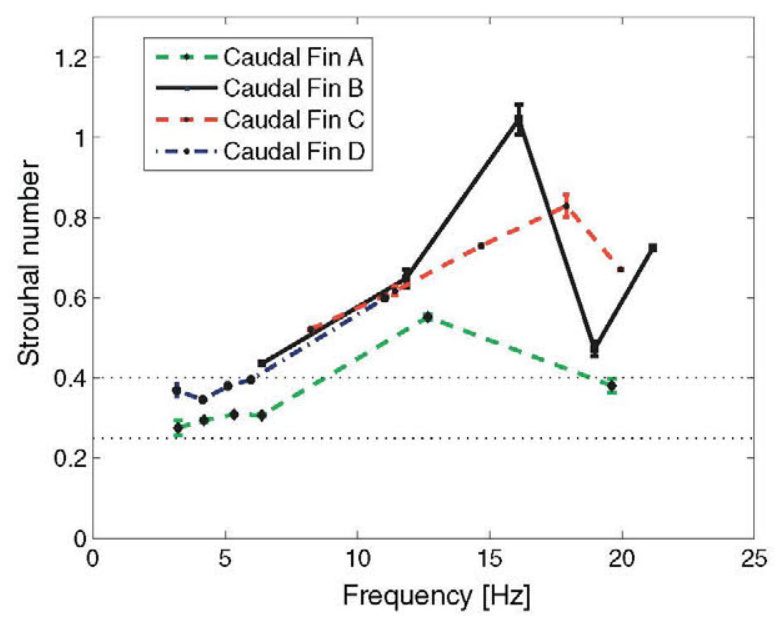

Fig. 24. Strouhal number vs, tail-beat frequency. 


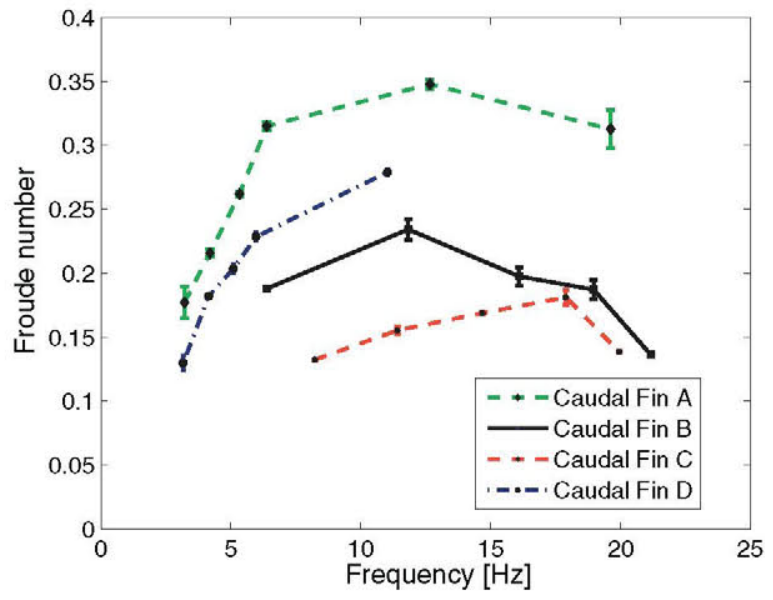

Fig. 25. Froude number vs. tail-beat frequency.

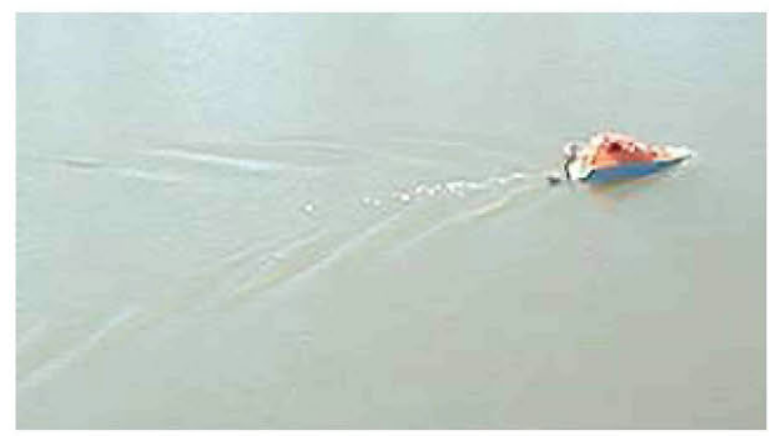

Fig. 26. Prototype during a field test.

where $g$ is the acceleration due to gravity $\left(9.8 \mathrm{~m} / \mathrm{s}^{2}\right)$ and $L$ is the characteristic length of the ship. The Froude number is a measure of maneuverability, and it represents the ratio of inertial force to gravity force in the flow [26]. Fig. 25 shows the experimentally obtained results for the different types of caudal fins. Vertical bars represent the standard deviation. It can be seen that the caudal fin of type A exhibits a better maneuverability than the rest of caudal fins.

Fig. 26 proves that when the prototype is moving in a flat surface of water, a noticeable water edge is produced as also a significant disturbance of the mass of water behind the stern, which is a clear evidence of the high velocities obtained.

\section{Conclusions}

A mechanical actuator for the biomimetic propulsion of swimming devices and the experimental study of the effect of the caudal fin elasticity on the overall performance have been presented in this paper. In the proposed drive, the motor works at constant speed. Such an operation mode is the most efficient, because all motor power is spent only for the motion of the caudal fin.

Laboratory tests, carried out for various caudal fins, showed that the samples should be made of elastic materials and with a variable thickness. Caudal fin of type A was made taking into account these considerations and showed to be the best of all the tested caudal fins. In this kind of caudal fin the stiffness decreases simultaneously from the fixed end to the free end and from the top and bottom edges to the middle part. Therefore, it is shown that a caudal fin has better properties when its rigidity varies in two directions: relative to the vertical cross-section and relative to the horizontal cross-section. The stiffness variation relative to the vertical crosssection makes it possible to increase the efficiency of the operating member due to the fact that during operation in water the caudal fin bends and forces water backwards to a greater extent than to the side. The stiffness variation relative to the horizontal cross-section also increases the efficiency due to the fact that the operating member bends about the horizontal axis during its operation in water, which to a significant extent prevents water being forced upwards and downwards, and because of this increases the amount of water which is forced backwards. These two facts significantly increase the forwardly directed component of the force with which the caudal fin acts on the robot's body. Additional experiments were also addressed in order to determine the best opening angle of the caudal fins peduncles.

The noise generated by a caudal fin of type A was recorded using a hydrophone and was represented by means of a spectrogram. A comparison with the acoustic emissions from a propeller in different working conditions was also accomplished. The caudal fin demonstrated experimentally a less noisy operation than the propeller.

Finally, some experiments were performed in natural conditions. For these tests, the proposed actuator was installed in a small ship. The swimming speed, the thrust efficiency and the maneuverability were evaluated with the different caudal fins manufactured. Caudal fin of type A exhibited the highest velocities. The Strouhal number achieved with this type of caudal fin confirms that its performance is in the fast efficient swimming category. The Froude number also indicates its better maneuverability in comparison with the other types of caudal fins.

\section{Acknowledgements}

The authors acknowledge partial funding of this research under Robocity2030-II S2009/DPI-1559. Dr. A. Apalkov acknowledges the financial support from Ministry of Science and Innovation of Spain under Juan de la Cierva Programme. Dr. R. Fernández acknowledges the support received from CSIC under JAE-Doc Programme.

\section{References}

[1] S. Gutreuter, J.M. Dettmers, D.H. Wahl, Estimating mortality rates of adult fish from entrainment through the propellers of river towboats, Transactions of the American Fisheries Society 132 (4) (2003) 646-661

[2] D. Zhang, L. Wang, J. Yu, M. Tan, Coordinated transport by multiple biomimetic robotic fish in underwater environment, IEEE Transactions on Cont rol Systems Technology 15 (4) (2007) 658-671.

[3] M. Listak, G. Martin, D. Pugal, A. Aabloo, M. Kruusmaa, Design of a semiautonomous biomimetic underwater vehicle for environmental monitoring, in: 2005 IEEE International Symposium on Computational Intelligence in Robotics and Automation, 2005, pp. 9-14.

[4] J. Shao, L. Wang, J. Yu, Development of an artificial fish-like robot and its application in cooperative transportation, Control Engineering Practice 16 (2008) 569-584.

[5] D. Zhang, D. Hu, L. Shen, H. Xie, A bionic neural network for fish-robot locomotion, Journal of Bionic Engineering 3 (2006) 187-194.

[6] H. Chen, Z. Chang-an, Y.Xie-zhen, X. Xiao-zheng, C. Gang, Hydrodynamic analy sis and simulation of a swimming bionic robot tuna, Journal of Hydrodynamics 19 (2007) $412-420$.

[7] Z. Wang, G. Hang, J. Li, YangweiWang, K. Xiao, A micro-robot fish with embed ded sma wire actuated flexible biomimetic fin, Sensors and Actuators A: Physical 144 (2008) 354-360.

[8] C. Zhou, M. Tan, N. Gu, Z. Cao, S. Wang, L. Wang, The design and implementation of a biomimetic robot fish, International Journal of Advanced Robotic Systems 5 (2) (2008) 185-192.

[9] J. Liu, H. Hu, A $3 d$ simulator for autonomous robotic fish, International Journal of Automation and Computing 1 (2004) 42-50.

[10] J. Yu, et al., Motion control algorithms for a free-swimming biomimetic robot fish, Acta Automatica Sinica 31 (4) (2005) 537-542.

[11] G.V. Lauder, E.D. Tytell, Hydrodynamics of undulatory propulsion, Fish Physiology 23 (2005) 425-468.

[12] J. Yu, M. Tan, S. Wang, E. Chen, Development of a biomimetic robotic fish and its control, IEEE Transactions on Systems, Man and Cybernetics 34 (4) (2004) $1798-1810$.

[13] K.A. Morgansen, V. Duindam, R.J. Mason, J.W. Burdick, R.M. Murray, Nonlinea control methods for planar carangiform robot fish locomotion, in: Proceedings of the 2001 IEEE International Conference on Robotics \& Automation, Korea, 2001 
[14] Q. Yan, Z. Han, S. Zhang, J. Yang, Parametric research of experiments on a carangiform robotic fish, Journal of Bionic Engineering 5 (2008) 95-101.

[15] ]. Liu, H. Hu, Biological ins piration: from carangiform fish to multi-joint robotic fish, Journal of Bionic Engineering 7 (2010) 35-48.

[16] J.-k.L. Ying-xiang Liu, Wei-shan Chen, Research on the swing of the body of two-joint robot fish, Journal of Bionic Engineering 5 (2008) 159-165.

[17] M. Sfakiotakis, D.M. Lane, J.B.C. Davies, Review of fish swimming modes for aquatic locomotion, Journal of Oceanic Engineering 24 (2) (1999) 237-252.

[18] K. Low, Modelling and parametric study of modular undulating fin rays for fish robots, Mechanism and Machine Theory 44 (2009) 615-632.

[19] D. Zhang, D. Hu, L. Shen, H. Xie, Design of an artificial bionic neural network tocontrol fish-robot's locomotion, Neurocomputing 71 (2008) 648-654.

[20] S. Heo, T. Wiguna, H.C. Park, N.S. Goo, Effect of an artificial caudal fin on the performance of a biomimetic fish robot propelled by piezoelectric actuators, Journal of Bionic Engineering 4 (2007) 151-158.

[21] H.H. Jindong Liu, Ian Dukes, Novel mechatronics design for a robotic fish, in: IEEE/RSJ International Conference on Intelligent Robots and Systems, 2005, pp. 2077-2082.

[22] P.E. Sitorus, Y.Y. Nazaruddin, E. Leksono, A. Budiyono, Design and implementation of paired pectoral fins locomotion of labriform fish applied to a fish robot, Journal of Bionic Engineering 6 (2009) 37-45.

[23] I.N. Odintsev, V.D. Sizarev, I.A. Razumovskiy, A.A. Apalkov, Holographic interferometry for the modal analysis of the structural elements in a liquid, in: Proc. 8th International Conference on Flow-induced Vibration, vol. 1, Paris, France 2004, pp. 301-306.

[24] M.S. Triantafyllou, G.S. Triantafyllou, R. Gopalkrishnan, Wake mechanics for thrust generation in oscillating foils, Physics of Fluids A 3 (1991) 2835-2837.

[25] G.S. Triantafyllou, M.S. Triantafyllou, M.A. Grosenbauch, Optimal thrust development in oscillating foils with application to fish propulsion, Journal of Fluids and Structures 7 (1993) 205-224.

[26] P.R. Bandyopadhyay, Trends in biorobotic autonomous undersea vehcles, IEEE Journal of Oceanic Engineering 30 (2005) 109-139.

\section{Biographies}

A. Apalkov was born in Moscow, Russia in 1966. He received the BS and the $\mathrm{PhD}$ degrees from the Department of Technical Physics at the National Research Nuclear
University "MEPhl", Moscow, in 1994 and 2005, respectively. Since 2009 he is a researcher at the Cent re for Automation and Robotics CSIC-UPM (Spanish National Research Council and Polytechnic University of Madrid). His scientific interests are related to all fields of experimental mechanics, with emphasis on implementing highly accurate optical interferometric measurements of local mechanical response.

R. Fernández received his $\mathrm{PhD}$ degree in industrial engineering from Polytechnic University of Madrid in 2006. She was a visiting student at the Center for Control Engineering and Computation at the University of California, Santa Barbara in 2003 and at the Department of Automatic Control at Lund University in 2004. She is cur rently a researcher at the Centre for Automation and Robotics CSIC-UPM (Spanish National Research Council and Polytechnic University of Madrid). Her research interests include design and control of field and service robots, nonlinear control theory, resonance and quasi-resonance drives and biomedical sensors.

J.G. Fontaine received his degrees from ENSET and an Advanced Studies Diploma in sensors and instrumentations at Orsay University (1983, Paris XI). He prepared his PhD's degree (1987) in robotics at LIMRO from the same University. In 1995, he received the Habilitation Diploma (DSc) from the Pierre et Marie Curie University (Paris VI) at L.R.P. (Laboratoire de Robotique de Paris). Currently, he is the Research Director of the Telerobotics and Applications Department at the Italian Institute of Technology. His main field of interest deals with teleoperation, telepresence and telexistence with mobile robots and micro and nano worlds exploration.

T. Akinfiev (1946-2010) received his MS degree from the Moscow State University and PhD degree from Mechanical Engineering Research Institute of the Academy of Sciences of Russia. From Year 1976 he was Researcher, Principal Researcher and Head of the Research Laboratory at the Mechanical Engineering Research Institute of the Academy of Sciences of Russia. From 1995 to 2010 he was a researcher at the Industrial Automation Institute (CSIC), and at the Centre for Automation and Robotics CSIC-UPM. His research interests included oscillation theory, mechanical engineering, control systems, robotics, intelligent drives, and mechat ronics.

M. Armada received his PhD in physics from the University of Valladolid (Spain) in 1979. He is Professor of Research at the CSIC (Spanish National Research Council) and Director of the Centre for Automation and Robotics. CAR (CSIC-UPM) where his main research direction is concentrated in robot design and control, with special emphas is in legged locomotion, agricultural robotics and humanitarian demining. 